\title{
Maturidade perceptomotora e reconhecimento de palavras: estudo correlacional entre o Bender - Sistema de Pontuação Gradual e o Teste de Reconhecimento de Palavras
}

\author{
Perceptive-motor maturity and word recognition: a \\ correlational study between Bender - Scoring \\ System for Grades and Word Recognition test
}

\author{
Laura de CARVALHO \\ Ana Paula Porto NORONHA ${ }^{2}$ \\ Lariana Paula PINTO²
}

Luana LUCA ${ }^{2}$

\begin{abstract}
Resumo
O presente estudo objetivou investigar evidências de validade para o Bender - Sistema de Pontuação Gradual, pela relação com o Teste de Reconhecimento de Palavras, partindo da premissa de que os testes apresentariam comunalidade em certos aspectos. Além disso, a pesquisa buscou verificar se o Bender é sensível para captar diferenças de média por idade. Participaram do estudo 297 crianças, sendo 153 do sexo masculino (51,5\%), com idade entre 7 e 10 anos ( $M=9,04, D P=0,88$ ). Cursavam do $2^{\circ}$ ao $5^{\circ}$ ano do ensino fundamental, em escola particular $(27,3 \%)$ ou pública $(72,7 \%)$ do interior do estado de São Paulo. Os instrumentos foram aplicados coletivamente, sendo que as figuras do Bender foram projetadas. Foram evidenciadas correlações significativas entre os instrumentos. Verificou-se também que o Bender mostrou-se sensível em captar as diferenças relacionadas à idade, revelando o aspecto maturacional avaliado pelo teste, assim como também revelou relação com a escolaridade.
\end{abstract}

Unitermos: Avaliação psicológica. Desenvolvimento humano. Teste de Bender-Gestalt.

\begin{abstract}
The aim of this study was to investigate evidence of validity of the Bender - Scoring System for Grades, by comparison with the Word Recognition Test, starting with the premise that the tests would show commonality in certain aspects. In addition, the study sought to verify whether the B-SPG is sensitive to capture differences in mean for age. The study participants included 297 children, 153 being boys (51.5\%) aged between 7 and 10 years $(M=9.04, S D=0.88)$. They were in the $2^{\text {nd }}$ to the $5^{\text {th }}$ year of elementary schooling, at private $(27.3 \%)$ and public (72.7\%) schools, in the interior of State of São Paulo. The instruments were applied collectively, and the Bender-Gradual Scoring System pictures were projected. Significant correlations were observed between the instruments. Furthermore, it was found that the Bender-Gradual Scoring System was shown to be sensitive in capturing differences related to age, revealing the aspect of maturity assessed by testing, and also revealed relationship with progress in school stages.
\end{abstract}

Unitems: Psychological assessment. Bender-Gestalt test. Human developmental.

$\boldsymbol{\nabla} \boldsymbol{\nabla} \boldsymbol{\nabla} \boldsymbol{\nabla}$

1 Universidade Federal de Mato Grosso, Instituto de Ciências Humanas e Sociais, Curso de Psicologia. Rodonópolis, MT, Brasil.

2 Universidade São Francisco, Programa de Pós-Graduação Stricto Sensu em Psicologia. R. Alexandre Rodrigues Barbosa, 45, Centro, 13251-900, Itatiba, SP, Brasil. Correspondência para/Correspondence to: L. PINTO. E-mail: <lariana_paula@hotmail.com>. 
Desde a sua construção e publicação, em 1938, diversos sistemas de correção foram desenvolvidos para oTeste Gestáltico Visomotor de Bender, permitindo-Ihe tanto uma análise qualitativa como quantitativa, de acordo com cada finalidade de avaliação: instrumento projetivo, avaliação da maturidade perceptomotora, diagnóstico de lesão cerebral ou transtornos mentais, dentre outros (Nunes, Ferreira \& Lopes, 2007). Ao desenvolver o instrumento, Lauretta Bender teve como objetivo estudar a função motriz da estruturação visual, a partir da mensuração de sua maturação. Com base nos trabalhos de Wertheimer, Bender (1955) selecionou nove, entre 30 figuras, que considerava mais apropriadas para a medida do nível maturacional da ação visomotora do indivíduo.

Contudo, Bender (1955) utilizava o instrumento com finalidade clínica, avaliando as respostas apresentadas pelas crianças apenas de maneira qualitativa, o que propiciou a elaboração de outras formas mais objetivas de interpretação e a realização de novas pesquisas por outros autores. No que concerne aos principais sistemas de correção criados, Field, Bolton e Dana (1982) destacaram que entre as décadas 50 e 60 foram desenvolvidos pelo menos oito sistemas. Os autores ainda ressaltaram que, em uma revisão da literatura realizada na década de 1960, mais de 130 obras sobre o Teste Bender foram publicadas. Aproximadamente, um quinto das publicações foi dedicado às crianças, principalmente depois de 1955, evidenciando uma preocupação crescente sobre o valor do instrumento para a faixa etária infantil.

Assim, o Teste de Bender, nos seus diversos sistemas, passou por amplos estudos, alguns relacionados às propriedades psicométricas (Engin \& Wallbrown, 1976; Fuller \& Wallbrown, 1983; Furr, 1970; Kroeff, 1988; Mehlman \& Vatovec, 1956; Pinelli \& Pasquali, 1991/1992; Rajabi, 2009; Soto, 2009), outros voltados à sua relação com outros construtos, com especial destaque para os processos de leitura e escrita (Duffy, Glair, Egeland \& Dinello, 1972; Giebink \& Birch, 1970; Keogh, 1965; Koppitz, 1975; Machado, 1978; Mazzeschi \& Lis, 1999; Nielsen \& Sapp, 1991; Stedman, Lawlis, Cortner \& Achterberg, 1978; Suehiro \& Santos, 2006; Vendemiatto, 2007). Sob esse aspecto, diversos autores têm relacionado a leitura e escrita ao desenvolvimento perceptomotor, partindo

372 do pressuposto de que o desenvolvimento adequado seria um dos requisitos para a aquisição de diversas habilidades e, especialmente, as de leitura e escrita, conforme já preconizado por Bender (1955).

Há 20 anos, Salvia e Ysseldyke (1991) afirmaram que o teste Bender já era muito utilizado com crianças, sendo indicado para predição de desempenho escolar, diagnóstico de problemas de leitura e escrita, além da avaliação de problemas emocionais e suspeita de lesão cerebral, entre outros. No Brasil, dentre os sistemas mais conhecidos e aplicados na população infantil, destaca-se o Developmental Bender Test Scoring System, criado por Koppitz (1975); tendo como objetivo avaliar a maturidade neurológica da criança (Koppitz, 1989). Entretanto, alguns estudos apontaram fragilidades quando ele era aplicado à população brasileira (Moura, 1982; La Puente \& Maciel Jr., 1984; Pinelli Jr. \& Pasquali, 1991/1992; Sisto, Noronha \& Santos, 2004; Sisto, Santos \& Noronha, 2004).

Considerando a importância do Teste Gestáltico de Bender, seu histórico de inúmeras pesquisas e os estudos psicométricos com resultados não favoráveis para aplicação e uso na população brasileira, Sisto, Noronha e Santos (2005) desenvolveram nova proposição de avaliação para ser utilizada com crianças. Nesse sentido, foi construído um sistema de aplicação e de correção adaptado às necessidades de normatização com amostra brasileira, o Bender - Sistema de Pontuação Gradual (B-SPG). Pesquisas realizadas têm apontado sua sensibilidade para captar a maturidade perceptomotora, bem como habilidades específicas associadas em diferentes grupos (Bartholomeu, 2006; Neri, 2005; Noronha, Santos \& Sisto, 2007; Rueda, Suehiro \& Silva, 2008; Sisto, Santos \& Noronha, 2010; Suehiro, 2008; Suehiro \& Santos, 2006; Vendemiatto, 2007).

Dentre as pesquisas, merece destaque a de Suehiro e Santos (2005), cujo objetivo foi buscar evidência de validade de critério para o B-SPG, ao comparar grupos divididos por dificuldades de aprendizagem (acentuada/média/leve/sem indícios). Foram participantes do estudo 287 crianças, de ambos os gêneros, com idade entre 7 e 10 anos ( $M=8,6, D P=0,76)$, provenientes de escolas públicas e particulares. Os instrumentos utilizados foram um questionário de identificação dos sujeitos, a Escala de Avaliação de Dificuldades na Aprendizagem da Escrita (ADAPE) e as nove figuras B-SPG. Os resultados permitiram a identificação de evidências de validade para o B-SPG, visto que diferen- 
ciaram os grupos quanto aos níveis de dificuldade de aprendizagem. Além disso, as autoras concluíram que crianças com problemas na aprendizagem da escrita tendem a apresentar dificuldades perceptomotoras.

No ano seguinte, as mesmas autoras desenvolveram um estudo com a finalidade de averiguar outras evidências de validade para o B-SPG. Para tanto, analisaram 237 protocolos de crianças com média de idade de 8,48 anos ( $D P=0,501$ ), de ambos os gêneros, estudantes de escolas públicas e particulares, do terceiro e quarto anos. Os resultados indicaram o aumento do desempenho em razão do ano frequentado, diferenciando significativamente a pontuação, de modo que as crianças do quarto ano apresentaram maiores notas em relação às outras. Assim, as autoras concluíram que houve associação entre a maturidade perceptomotora e a fase de escolarização (Suehiro \& Santos, 2006).

Noronha et al. (2007) objetivaram buscar evidências de validade para o B-SPG, pela investigação das mudanças desenvolvimentais em função dos escores obtidos por idade. Fizeram parte do estudo 1052 crianças de escolas públicas, que cursavam do primeiro ao quinto ano do ensino fundamental, com média de idade de 8,35 anos (DP=1,29). As análises de correlação realizadas pelos autores indicaram valores moderados no que se refere ao escore e à idade, sendo negativo e significativo estatisticamente, indicando que, com a progressão da idade, os erros da cópia diminuem. Os autores confirmaram o caráter maturacional.

Em seu estudo, Suehiro (2008) teve por objetivos buscar evidências de validade entre instrumentos que se prestam à avaliação da compreensão em leitura, aprendizagem da escrita, desenvolvimento perceptomotor e consciência fonológica. Participaram 221 estudantes, de ambos os gêneros, entre 6 e 12 anos $(M=8,53$, $D P=1,40)$, do segundo ao quinto ano do ensino fundamental de uma escola pública. No que se refere aos resultados obtidos no desempenho no B-SPG, a autora encontrou diferenças significativas nas médias obtidas por idade e por ano escolar. Quando analisada a correlação entre os instrumentos, o B-SPG apresentou coeficientes moderados e significativos em relação a outros instrumentos utilizados na pesquisa, reforçando sua relação com as habilidades de leitura e escrita.
É possível observar que são poucas as pesquisas que se relacionam à leitura e à maturidade perceptomotora, quando avaliada pelo B-SPG. Assim, buscando ampliar os estudos com essa finalidade, o objetivo deste estudo foi verificar a relação da maturidade perceptomotora com a compreensão em leitura e escrita. Para tanto, procedeu-se à prova de Correlação de Pearson entre os instrumentos (B-SPG e Teste de Reconhecimento de Palavras), e destes com as variáveis idade e escolaridade.

\section{Método}

\section{Participantes}

Participaram do estudo 297 crianças, com idade variando entre 7 e 10 anos e média de 9,04 anos (DP=0,88), sendo 22 sujeitos com 7 anos (7,4\%), 43 sujeitos com 8 anos (14,5\%), 132 com 9 anos (44,4\%) e 100 com 10 anos de idade (33,7\%). Do total de participantes, 144 eram do sexo feminino (48,5\%) e 153 do masculino (51,5\%). No que se refere à escolaridade, $18(6,1 \%)$ cursavam o $2^{\circ}$ ano; $24(8,1 \%)$ estavam matriculados no $3^{\circ}$ ano; 118 $(39,7 \%)$ frequentavam o $4^{\circ}$ ano, e 137 (46,5\%) estavam no $5^{\circ}$ ano. Do total, 72,7\% estudavam em escola pública, e 27,3\%, em particular. Foram convidados a participar todas as crianças das salas de aula, não tendo havido controle de variáveis, como por exemplo, a repetência escolar.

\section{Instrumentos}

Os instrumentos utilizados para a realização do estudo foram os Testes Gestáltico Visomotor de B-SPG e o Teste de Reconhecimento de Palavras, abaixo descritos.

Teste Gestáltico Visomotor de Bender: Sistema de Pontuação Gradual (B-SPG) (Sisto et al., 2005). O B-SPG avalia a maturidade perceptomotora, por meio da análise da distorção da forma, pela reprodução à mão livre de nove figuras. Essa distorção, como critério de análise, é entendida como o desrespeito aos aspectos estruturais do desenho, de modo que pontos, linhas, retas, curvas e ângulos são desenhados sem precisão. Assim, os erros são pontuados, atribuindo-se uma nota de 0 a 3 pontos para cada figura, conferindo uma pontuação total que varia de 0 a 21. O teste é destinado a crianças de 6 a 10 
anos, com tempo de aplicação não superior a 15 minutos, embora não haja limite de tempo para a reprodução.

Estudos psicométricos já foram efetivados com o B-SPG, em uma amostra de 1052 crianças, com média de idade de 8,35 anos e DP de 1,29. Das crianças estudadas, $51,1 \%$ eram do gênero feminino e 48,7\% do masculino, e todas frequentavam do $1^{\circ}$ ao $5^{\circ}$ anos do ensino fundamental. Nos estudos para evidências de validade baseada nas mudanças desenvolvimentais, encontrou-se a existência de correlação entre a idade (6 a 10 anos) e pontuação, com coeficiente de $r=-0,58$, $(p<0,001)$. Os resultados revelaram que, com o aumento da idade, houve uma diminuição progressiva das distorções de forma e, consequentemente, dos erros. Em análises para verificação da consistência interna, o teste apresentou bons índices (alfa de Cronbach 0,80; Spearman-Brown 0,77 e modelo Rasch 0,76).

Em relação às normas de interpretação dos resultados, estas são fornecidas em relação a duas medidas. Uma delas é referente à pontuação bruta, produto da avaliação dos protocolos pela aplicação direta dos critérios de correção considerando os erros; e a outra, pela medida de Rasch para análises que tenham necessidade de uma informação dessa natureza.

Teste do Reconhecimento de Palavras (Kingesk \& Sisto, 2004). Consiste em um teste de múltipla escolha, com 55 itens de três alternativas de resposta, das quais somente uma é correta. São palavras que foram organizadas em ordem alfabética, e para cada uma delas foram criadas outras duas com erros gramaticais. $O$ estudo realizado por Kingeski e Sisto (2004) revelou que a média de acertos do gênero feminino apresentou-se superior em relação ao masculino. A análise da variância entre as médias indicou diferença estatisticamente significativa entre as séries do ensino fundamental $(p<0,001)$, separando os escores das crianças nos grupos correspondentes ao tempo de escolaridade, e conferindo validade de critério ao instrumento. No Reconhecimento de Palavras, a cada acerto foi atribuído um ponto, sendo possível atingir o escore máximo de 55 pontos.

\section{Procedimento}

O projeto foi aprovado pelo Comitê de Ética em

374 Pesquisa da Universidade São Francisco, sob o Protocolo
CAAE 0059.0.142.000-07, em 14 de agosto de 2007, e, após, foi feito contato com a direção das instituições de ensino para apresentar o objetivo do estudo. Todos os pais ou responsáveis pelas crianças participantes do estudo assinaram o Termo de Consentimento Livre e Esclarecido antes de sua inclusão na amostra.

A aplicação ocorreu no próprio ambiente escolar, coletivamente nas classes, com grupos de no máximo 25 crianças. O tempo médio de aplicação dos dois instrumentos foi de aproximadamente 40 minutos, sendo que o Teste de Reconhecimento de Palavras foi o primeiro a ser aplicado. A fim de obter informações acerca dos dados demográficos dos participantes, as pesquisadoras elaboraram uma lista na qual constava a idade, o sexo e a série frequentada por cada criança.

\section{Resultados}

Tendo em vista os objetivos pretendidos neste estudo, inicialmente serão apresentadas as estatísticas descritivas dos instrumentos em questão, organizadas por idade. Na sequência dar-se-á a análise das correlações entre B-SPG e Teste de Reconhecimento de Palavras, bem como destes com as variáveis idade e escolaridade. Por fim será apresentada a comparação das médias e agrupamento por idade.

NaTabela 1, podem ser observados os resultados referentes ao desempenho dos participantes em ambos os instrumentos, por idade.

Verificou-se que, à medida que idade avançou, ocorreu uma diminuição na média de erros no B-SPG. O mínimo de erros apresentados foi 0 e o máximo 20, obtido pelas crianças de 9 anos (Tabela 1).

Buscando verificar a relação entre os construtos dos instrumentos do estudo, foi realizada a prova de correlação de Pearson (Tabela 2). Os coeficientes encontrados evidenciaram que todas as correlações foram significativas no nível de 0,001. Como esperado, o B-SPG apresentou correlação negativa com o Reconhecimento de Palavras ( $r=-0,29 ; p=0,000)$, visto que o primeiro pontua as distorções, e o segundo, os acertos. $\mathrm{O}$ índice obtido apresentou-se com magnitude baixa. Os resultados confirmam que os construtos estudados estão relacionados. Por outro lado, não se pode afirmar que tratem de um único domínio teórico, tendo em vista que o coeficiente de correlação foi baixo. 
Tabela 1. Estatística descritiva dos instrumentos por idade. Campinas (SP), 2007.

\begin{tabular}{|c|c|c|c|c|c|c|}
\hline Idade & Teste & $n$ & Mínimo & Máximo & Média & Desvio-Padrão \\
\hline \multirow[t]{2}{*}{7} & B-SPG & 22 & 2 & 18 & 9,14 & 3,745 \\
\hline & Reconhecimento de Palavra & 22 & 10 & 49 & 30,05 & 10,764 \\
\hline \multirow[t]{2}{*}{8} & B-SPG & 43 & 2 & 1 & 8,86 & 4,057 \\
\hline & Reconhecimento de Palavra & 43 & 0 & 55 & 35,51 & 12,115 \\
\hline \multirow[t]{2}{*}{9} & B-SPG & 132 & 0 & 20 & 7,06 & 3,673 \\
\hline & Reconhecimento de Palavra & 132 & 12 & 55 & 38,30 & 10,556 \\
\hline \multirow[t]{2}{*}{10} & B-SPG & 100 & 0 & 14 & 6,79 & 3,304 \\
\hline & Reconhecimento de Palavra & 100 & 11 & 55 & 41,27 & 10,354 \\
\hline
\end{tabular}

B-SPG: Bender - Sistema de Pontuação Gradual.

Tabela 2. Correlação de Pearson entre o Teste de B-SPG, o Teste de Reconhecimento de Palavras e as variáveis Idade e escolaridade. Campinas (SP), 2007.

\begin{tabular}{lccc}
\hline & & B-SPG & Reconhecimento de Palavras \\
\hline Idade & $r$ & $-0,207$ & 0,267 \\
Escolaridade & $p$ & 0,000 & 0,000 \\
& $r$ & $-0,135$ & 0,318 \\
Reconhecimento & $p$ & 0,020 & 0,000 \\
de Palavras & $p$ & $-0,29$ & \\
\hline
\end{tabular}

B-SPG: Bender - Sistema de Pontuação Gradual.

Tabela 3. Prova de Tukey para o teste de B-SPG na amostra geral por idade. Campinas (SP), 2007.

\begin{tabular}{lcccc}
\hline \multirow{2}{*}{ Idades } & $\mathrm{n}$ & \multicolumn{3}{c}{ B-SPG } \\
\cline { 3 - 5 } & & 1 & 2 & 3 \\
\hline 10 & 100 & 6,79 & & \\
9 & 132 & 7,06 & 7,06 & \\
8 & 43 & & 8,86 & 8,86 \\
7 & 22 & & & 9,14 \\
\hline
\end{tabular}

B-SPG: Bender - Sistema de Pontuação Gradual.

A análise correlacional também foi realizada para verificar a relação entre os construtos e as variáveis idade e escolaridade (Tabela 2). Os valores foram significativos, embora também com magnitudes baixas. No que se refere ao B-SPG, a correlação com a idade mostrou-se maior que com a escolaridade, embora ambas sejam significativas.

A fim de verificar se as médias do B-SPG se diferenciaram em relação às idades, foram realizadas a Análise de Variância (ANOVA) e a prova de Tukey (nível de significância de 0,05). A ANOVA revelou que a pontuação variou significamente entre as idades $[F(3,294)=5,359$; $p<0,001]$. Houve agrupamento das idades em relação à pontuação no Bender, em três conjuntos, conforme apresentado na Tabela 3. Tal achado indica que as crianças de 10 anos pontuam menos que crianças mais novas, como as de 7 anos por exemplo, e reforça a ideia de que a distorção da forma diminui com o avanço da idade. Além disso, as crianças de 8 e 9 anos da presente amostra tiveram desempenhos semelhantes, como observado naTabela 3.

\section{Discussão}

Tendo em vista as análises realizadas, e tendo como instrumento-foco o B-SPG, pode-se observar que os resultados obtidos aproximam-se daqueles encontrados nos estudos Suehiro e Santos (2005), Noronha et al. (2007) e Suehiro (2008). Adicionalmente, considerando a distribuição por idade, também foi verificado que as crianças mais velhas obtiveram melhores desempenhos que as mais novas, revelando consonância com os estudos de Machado (1978) e Mazzeschi e Lis (1999).

Quando analisadas as correlações, inicialmente com o Teste do Reconhecimento de Palavras, em que pese a escrita, pesquisas também revelaram a correlação da maturação e do nível intelectual como um fator que influencia o seu desenvolvimento. A literatura aponta que as crianças mais velhas apresentam melhor desempenho do que as mais novas em um mesmo nível escolar, considerando que, com o passar do tempo, a tendência é que haja maturação da habilidade perceptomotora (Machado, 1978; Mazzeschi \& Lis, 1999). No que diz respeito à idade e escolaridade, os índices obtidos evidenciam o caráter evolutivo do B-SPG e sua relação com as etapas iniciais acadêmicas. Tais achados estão 
em consonância com os estudos de Noronha, et al. (2007) e Suehiro (2008), no que concerte à idade, e ao de Suehiro e Santos (2005), à escolaridade.

Tendo por base as diferenças entre os desempenhos por idade, o que portanto traduz que crianças mais velhas apresentam menos erros que as mais novas, verificou-se que tal ocorrência se dá de maneira significativa, fortalecendo o aspecto desenvolvimental também avaliado pelo B-SPG. Os estudos de Noronha et al. (2007) e Suehiro (2008) também constataram esse aspecto. Além disso, algumas diferenças já haviam sido preconizadas pela autora original, que afirmava que as reproduções melhoraram com o aumento da idade, revelando o aspecto maturacional da habilidade perceptomotora (Bender, 1955). Algumas pesquisas também apontaram tendências para esse fenômeno quando estudados outros sistemas de correção do mesmo instrumento (Bolen, 2003; Garvey \& Popplestone, 1960; Rajabi, 2009).

Diante desses resultados, tomando como ponto de partida o fato de que o desenvolvimento das crianças dá-se a partir de suas experiências sensório-motoras nos primeiros anos de vida e, conforme Bender (1955), a maturidade perceptomotora é um requisito para o desenvolvimento das habilidades acadêmicas, dentre elas a leitura e a escrita; e considerando que a avaliação das dificuldades de aprendizagem constitui uma necessidade crescente, estruturou-se o objetivo do presente estudo, que foi verificar a correlação da maturidade perceptomotora com a compreensão em leitura e escrita, bem como a relação delas com a variável idade.

\section{Considerações Finais}

Os resultados obtidos colaboraram para a confirmação de que o Teste de Bender pode ser utilizado como um instrumento de avaliação, não somente para verificar a maturidade perceptomotora, mas também como mais um recurso para um amplo processo de avaliação, em especial, a avaliação da aprendizagem de leitura e escrita. Além disso, os estudos com o novo sistema de correção foram necessários, pois embora ele esteja em consonância com os requisitos ressaltados pelo Conselho Federal de Psicologia, no que se refere ao uso e comercialização, pesquisas com enfoques e amostras distintas
Entende-se que outros estudos deverão ser realizados e, da mesma forma, poderão contribuir com dados relevantes na área de avaliação e com o fomento de novas pesquisas no campo da aprendizagem. Para tanto, os instrumentos utilizados devem ser apropriados e coerentes com a realidade vivenciada pelas crianças brasileiras e pautados em princípios científicos. A esse respeito, são importantes os estudos que se proponham a verificar se de fato os instrumentos que estão sendo empregados medem o que se propõem a medir, considerando as populações específicas para as quais foram construídos, e quão bem o fazem.

Convém destacar que este estudo teve como referência uma amostra por conveniência e que, sob essa perspectiva, não foi possível controlar algumas variáveis relevantes - dentre as quais se as crianças possuíam algum tipo de dificuldade de aprendizagem, ou ainda, um número de distribuição equitativo dos participantes por idade, gênero e escolaridade. Isso posto, seria importante que outros estudos controlassem tais variáveis.

\section{Referências}

Bartholomeu, D. (2006). Teste Gestáltico Visomotor de Bender e Desenho da Figura Humana: convergências de avaliação? Dissertação de mestrado não-publicada, Universidade São Francisco, Itatiba.

Bender, L. (1955). Test Gestáltico Visomotor: usos y aplicaciones clinicas ( $6^{a}$ ed.). Buenos Aires: Paidós.

Bolen, L. M. (2003). Constructing local age norms based on ability for the Bender-Gestalt Test. Perceptual and Motor Skills, 97 (2), 467-476.

Duffy, O. B., Glair, T. N., Egeland, B., \& Dinello, A. D. (1972). Relationship of intelligence, visual-motor skills, and psycholinguistic abilities with achievement in the third, fourth, and fifth grades: a follow-up study. Journal of Educational Psychology, 63 (4), 358-362.

Engin, A. W., \& Wallbrown, F. H. (1976). The stability of four kinds of perceptual errors on the Bender-Gestalt. The Journal of Psychology, 94 (1), 123-126.

Field, K., Bolton, B., \& Dana, R. H. (1982). An evaluation of three Bender-Gestalt scoring systems as indicators of psychopathology. Journal of Clinical Psychology, 38 (4), 838-842.

Fuller, G. B., \& Wallbrown, F. H. (1983). Comparison of the Minnesota Percepto-Diagnostic Test and Bender Gestalt: relationship with achievement criteria. Journal of Clinical Psychology, 39 (6), 985-988.

Furr, K. D. (1970). Standard scores for the Koppitz Developmental Scoring System. Journal of Clinical Psychology, 26 (1), 78-79. 
Garvey, M. J., \& Popplestone, J. A. (1960). Influence of age and sex on Bender Gestalt associations. Perceptual and Motor Skills, 11 (3), 258.

Giebink, J. W., \& Birch, R. (1970). The Bender Gestalt Test as an ineffective predictor of reading achievement. Journal of Clinical Psychology, 26 (4), 484-485.

Keogh, B. A. (1965). The Bender Gestalt as a Predictive and Diagnostic Test of Reading Performance. Journal of Consulting Psychology, 29 (1), 83-84.

Kingeski, M. F., \& Sisto, F. F. (2004). Reconhecimento de palavras nas séries iniciais do ensino fundamental. Teoria e Prática da Educação, 7 (2), 183-190.

Koppitz, E. M. (1975). Bender Gestalt Test, Visual Aural Digit Span Test and Reading Achievement. Journal of Learning Disabilities, 8 (3), 32-35.

Koppitz, E. M. (1989). OTeste Gestáltico de Benderpara crianças. Porto Alegre: Artes Médicas.

Kroeff, P. (1988). Normas brasileiras para o Teste de Bender. Psicologia: Reflexão e Crítica, 1/2 (3), 12-19.

La Puente, M., \& Maciel Jr., J. (1984). Procedimentos operacionais na avaliação do Teste de Bender Infantil. Estudos de Psicologia, 3 (4), 76-92.

Machado, M. C. L. (1978). Uso do Teste de Benderpara avaliar a organização perceptiva motora de escolares paulistas. Dissertação de mestrado não-publicada, Pontifícia Universidade Católica de São Paulo.

Mazzeschi, C., \& Lis, A. (1999). The Bender-Gestalt test: Koppitz's Developmental Scoring System administered to two samples of Italian preschool and primary school children. Perceptual and Motor Skills, 88 (3Pt2), 1235-1244.

Mehlman, B., \& Vatovec, E. (1956). A Validation Study of the Bender-Gestalt. Journal of Consulting Psychology, 20 (1), 71-74.

Moura, M. L. S. (1982). Reconhecimento e reprodução de figuras do Teste Gestáltico Visomotor de Bender: um estudo evolutivo. Arquivos Brasileiros de Psicologia, 34 (4), 50-61.

Neri, M. L. (2005). Bender - Sistema de Pontuação Gradual: um estudo com crianças surdas. Dissertação de mestrado não-publicada, Universidade São Francisco, Itatiba.

Nielsen, S., \& Sapp, G. L. (1991). Bender Gestalt developmental scores: predicting reading and mathematics achievement. Psycological Reports, 69 (1), 39-42.

Noronha, A. P. P., Santos, A. A. A., \& Sisto, F. F. (2007). Evidências de validade do Bender: sistema de pontuação gradual. Psicologia: Reflexão e Crítica, 20 (2), 335-341.

Nunes, M. L. T., Ferreira, R. B., \& Lopes, F. (2007). Diferentes sistemas de aplicação e interpretação do Teste Gestáltico Visomotor de Bender. Psic: Revista de Psicologia da Vetor Editora, 8 (1), 41-49.
Pinelli Jr., B., \& Pasquali, L. (1991/1992). Parâmetros psicométricos do Teste Gestáltico Visomotor de Bender: um estudo empírico. Revista de Psicologia, 1/2 (9/10), 51-74.

Rajabi, G. (2009). Normalizing the Bender Visual-Motor Gestalt Test among 6-10 year-old children. Journal of Applied Sciences, 9 (6), 1165-1169.

Rueda, F. J. M., Suehiro, A. C. B., \& Silva, M. A. (2008). Precisão entre avaliadores e pelo método teste-reteste no Bender: sistema de pontuação gradual. Psicologia:Teoria e Prática, 10 (1), 25-35.

Salvia, J., \&Ysseldyke, J. (1991). Avaliação em educação especial e corretiva. São Paulo: Manole.

Sisto, F. F., Noronha, A. P. P., \& Santos, A. A. A. (2004). Distorção de forma no teste de Bender: questionado seu critério de validade. Revista do Departamento de Psicologia da UFF, 16 (2), 139-154.

Sisto, F. F., Noronha, A. P. P, \& Santos, A. A. A. (2005). Manual Bender: sistema de pontuação gradual. São Paulo: Vetor Editora Psicopedagógica.

Sisto, F. F., Santos, A. A. A., \& Noronha, A. P. P. (2004). Critério de integração do Teste de Bender: explorando evidências de validade. Avaliação Psicológica, 3 (1) 13-20.

Sisto, F. F., Santos, A. A. A., \& Noronha, A. P. P. (2010). Differential Functioning of Bender Visual-Motor Gestalt Test Items. Perceptual and Motor Skills, 110 (1), 313-322.

Soto, C. M. (2009). Un análisis no paramétrico de ítems de la prueba del Bender modificado para estudiantes de primaria. Liberabit, 15 (2), 83-94.

Stedman, J. M., Lawlis, G. F., Cortner, R. H., \& Achterberg, G. (1978). Relationships between WISC-R factors, WideRange Achievement Test Scores, and visual-motor maturation in children referred for psychological evaluation. Journal of Consulting and Clinical Psychology, 46 (5), 869-872

Suehiro, A. C. B. (2008). Processos fonológicos e perceptuais e aprendizagem da leitura e escrita: instrumentos de avaliação. Tese de doutorado não-publicada, Universidade São Francisco, Itatiba.

Suehiro, A. C. B., \& Santos, A. A. A. (2005). O Bender e as dificuldades de aprendizagem: estudo de validade. Avaliação Psicológica, 4 (1), 23-31.

Suehiro, A. C. B., \& Santos, A. A. A. (2006). Evidência de validade de critério do Bender: sistema de pontuação gradual. Interação em Psicologia, 10 (2), 217-224.

Vendemiatto, B. C. (2007). Medidas de habilidades cognitiva e visomotora: evidências de validade do Bender-SPG. Dissertação de mestrado não-publicada, Universidade São Francisco, Itatiba.

Recebido em: 23/5/2011

Versão final reapresentada em: 20/10/2011

Aprovado em: 4/11/2011 
